\title{
Evaluation of malignancy using Ki-67 labeling index for gastric stromal tumor
}

\author{
Yoshinide Nagasako ${ }^{1}$, Kazuhito Misawa ${ }^{1}$, Shigechika Kohashi ${ }^{1}$, Kimiharu Hasegawa ${ }^{1}$, Yumi Okawa ${ }^{1}$, \\ Hidekazu SAno ${ }^{1}$, Akio Takada ${ }^{2}$, and Hidetoshi Sato ${ }^{2}$ \\ ${ }^{1}$ Department of Surgery, Sapporo City General Hospital, Kita-11 Nishi-13, Chuo-ku, Sapporo 060-8604, Japan \\ ${ }^{2}$ Department of Pathology, Sapporo City General Hospital, Sapporo, Japan
}

\begin{abstract}
Background. Assessment of malignant potential in gastrointestinal stromal tumors (GISTs) is still problematic. The maximum tumor diameter and the mitotic index are generally used as an index of malignancy of GISTs. The Ki-67 labeling index has recently been used as an index of cell growth, and the prognosis of GISTs was reported to be significantly poor when the value of this index was $10 \%$ or higher.

Methods. Clinicopathological and immunohistological factors were analyzed in 15 patients who underwent surgical resection of gastric stromal tumors at our department between April 1997 and July 2002. The patients were divided into "metastasis/recurrence" and "benign" groups. Also, the relationship of changes in the Ki-67 labeling index to the degree of malignancy in recurrent lesions was assessed in an 84-year-old woman who underwent five reoperations because of recurrences in the peritoneum.

Results. Significant differences were noted between the metastasis/recurrence and benign groups in relation to the mean maximum tumor diameter $(186.7 \pm 80.8 \mathrm{~mm}$ vs $41.3 \pm$ $22.9 \mathrm{~mm})$, mitotic index (88.3 $\pm \mathbf{5 . 0 / 5 0}$ high-power fields [HPF] vs 3.0 \pm 2.9/50 HPF), and the Ki-67 labeling index $(11.4 \pm 2.5 \%$ vs $0.01 \pm 0.51 \%)$. In the patient who had metastasis to the liver 3.5 years after initial operation and underwent five reoperations before death, the intervals until detection of recurrence tended to be shortened gradually. The Ki-67 labeling index varied with each operation, and tended to be higher at the time of reoperations than at the initial operation.

Conclusion. The maximum tumor diameter, mitotic index, and Ki-67 labeling index were useful as an index of malignancy for gastric stromal tumor. The efficacy of surgical resection alone may be insufficient in patients with disseminated metastasis to the peritoneum.
\end{abstract}

Key words Gastric stromal tumor · Ki-67 labeling index

Offprint requests to: Y. Nagasako

Received: April 22, 2003 / Accepted: June 2, 2003

\section{Introduction}

The origin of gastrointestinal stromal tumor (GIST), the generic name for gastrointestinal mesenchymal tumors in a narrow sense, is said to be Cajal's interstitial cells that exist in the gastrointestinal muscle layer. It is important to objectively evaluate the biological malignancy of GIST, which is potentially malignant, but it is difficult to determine its malignancy by histology at present. The Ki-67 labeling index has recently been used as an excellent index of cell growth; the index is calculated by immunohistochemically evaluating the cell growth-related antigen Ki-67, using the monoclonal antibody MIB-1 [1]. In this article, the utility of the Ki-67 labeling index as an index of malignancy was assessed first, and then changes in malignancy were investigated in a patient with primary gastric stromal tumor who underwent a total of five reoperations because of recurrences in the peritoneum.

\section{Patients and methods}

Fifteen patients with gastric stromal tumors who underwent surgical resection at our department between April 1997 and July 2002 were retrospectively analyzed. Three patients who had metastasis at the time of the initial operation, or recurrence after operation, were classified as the metastasis/recurrence group, while 12 patients who were alive without postoperative recurrence were classified as the benign tumor group. As prognostic determinants, we examined age, sex, mean maximum tumor diameter, presence of hemorrhage from tumor, presence of tumor necrosis, and the pattern of macroscopic growth type, investigated by clinical pathology, while the mitotic index (the frequency of mitosis in ten visual fields at a magnification of $\times 400$ ), p53 expression, c-kit protein, CD34, alpha-smooth muscle actin (SMA), S-100 protein, desmin, vimentin, and the 
Ki-67 labeling index (the number of MIB-1-positive cells determined by counting 1000 cells at a magnification of $\times 400$ ) were examined by histopathology. In an 84-year-old woman in the metastasis/recurrence group who underwent a total of five reoperations because of recurrences in the peritoneum, changes in the malignancy of the gastric stromal tumor were assessed. At each operation, resected peritoneal tumors were examined in order to calculate the Ki-67 labeling index. The significance of differences was determined by the $\chi^{2}$ test or $t$-test, and the level of significance was set at $P<0.05$.

\section{Results}

The mean maximum diameter, mitotic index, and Ki-67 labeling index showed significant differences between the two groups. Briefly, the mean maximum tumor diameter was significantly larger in the metastasis/recurrence group $(186.7 \pm 80.8 \mathrm{~mm})$ than in the benign tumor group $(41.3 \pm 22.9 \mathrm{~mm})$, and the mitotic index was significantly higher in the metastasis/recurrence group $(88.3 \pm 5.0 / 50$ high-power fields $[\mathrm{HPF}])$ than in the benign tumor group $(3.0 \pm 2.9 / 50 \mathrm{HPF})$. The Ki-67 labeling index was also significantly higher in the metastasis/recurrence group $(11.4 \pm 2.5 \%)$ than in the benign tumor group $(0.01 \pm 0.51 \%)$ (Table 1$)$. Further, the mitotic index was significantly correlated with the Ki-67 labeling index $\left(r^{2}=0.93 ; P<0.001\right.$; Fig. 1$)$. When gastric stromal tumor was divided into types according to Rosai's immunohistological classification [2], the relative incidence was uncommitted type (11 patients) $>$ smooth muscle type ( 3 patients) $>$ neural type ( 1 patient) $>$ combined type (none), and no relationship to malignancy was noted. All patients were negative for p53 expression.

On June 19 1998, the patient under consideration underwent the first operation because of primary gastric stromal tumor. Subsequently, she underwent a total of five reoperations because of recurrences in the peritoneum (Fig. 2). She had metastasis to the liver 3.5 years after the initial operation and died of metastasis to the liver and peritoneum. The intervals until the detection of recurrence by abdominal computed tomography (CT) examination tended to decrease gradually, from 322 days to $192,77,51,72$, and 85 days. The Ki-67 labeling index of malignancy was $13.9 \%$ at the time of the initial operation, and it subsequently changed to $26.5 \%, 32.9 \%, 16.1 \%, 37.0 \%$, and $26.7 \%$ (Figs. 2 and 3 ). The Ki-67 labeling index, showing the malignancy of the gastric stromal tumor, varied with each operation, and tended to be higher at the time of reoperations than at the time of the initial operation.

\section{Discussion}

The diameter of the tumor and the mitotic index have been reported as useful indexes of the biological malig-

Table 1. Comparison of clinicopathological and immunohistochemical factors by presence of metastasis or recurrence of gastric stromal tumors

\begin{tabular}{lccc}
\hline & $\begin{array}{c}\text { Benign tumor } \\
\text { group }(n=12)\end{array}$ & $\begin{array}{c}\text { Metastasis/recurrence } \\
\text { group }(n=3)\end{array}$ & Statistical analysis \\
\hline Age (years; mean \pm SD) & $66.6 \pm 9.1$ & $69.0 \pm 13.7$ & NS \\
Sex (male/female) & $5 / 7$ & $2 / 1$ & NS \\
Size (mm; mean \pm SD) & $41.3 \pm 22.9$ & $186.7 \pm 80.8$ & $P<0.001$ \\
Macroscopic (Endo $/$ Exo/Intrac/Mixed $)$ & $1 / 3 / 5 / 3$ & NS \\
Ulcer formation (positive/negative) & $0 / 12$ & $1 / 2 / 0 / 0$ & NS \\
Hemorrhage (positive/negative) & $1 / 11$ & NS \\
Necrosis (positive/negative) & $0 / 12$ & NS \\
Mitotic index (/50 HPF; mean \pm SD) & $3.00 \pm 2.9$ & $0 / 3$ & $P<0.001$ \\
Ki-67 labeling index (percentage; mean \pm SD) & $0.01 \pm 0.51$ & $88.3 \pm 5.0$ & $P<0.001$ \\
c-kit protein (positive/negative) & $12 / 0$ & NS \\
CD34 (positive/negative) & $10 / 2$ & $3 / 0$ & NS \\
Vimentin (positive/negative) & $10 / 2$ & $3 / 0$ & NS \\
SMA (positive/negative) & $3 / 9$ & $0 / 3$ & NS \\
S-100 protein (positive/negative) & $0 / 12$ & $1 / 2$ & NS \\
Desmin (positive/negative) & $2 / 10$ & $0 / 3$ & NS \\
p53 protein (positive/negative) & $0 / 12$ & $0 / 3$ & NS \\
\hline
\end{tabular}

HPF, high-power fields; SMA, smooth muscle actin; NS, not significant

${ }^{a}$ Endoluminal

${ }^{\mathrm{b}}$ Exoluminal

${ }^{\mathrm{c}}$ Intramural

dixed 


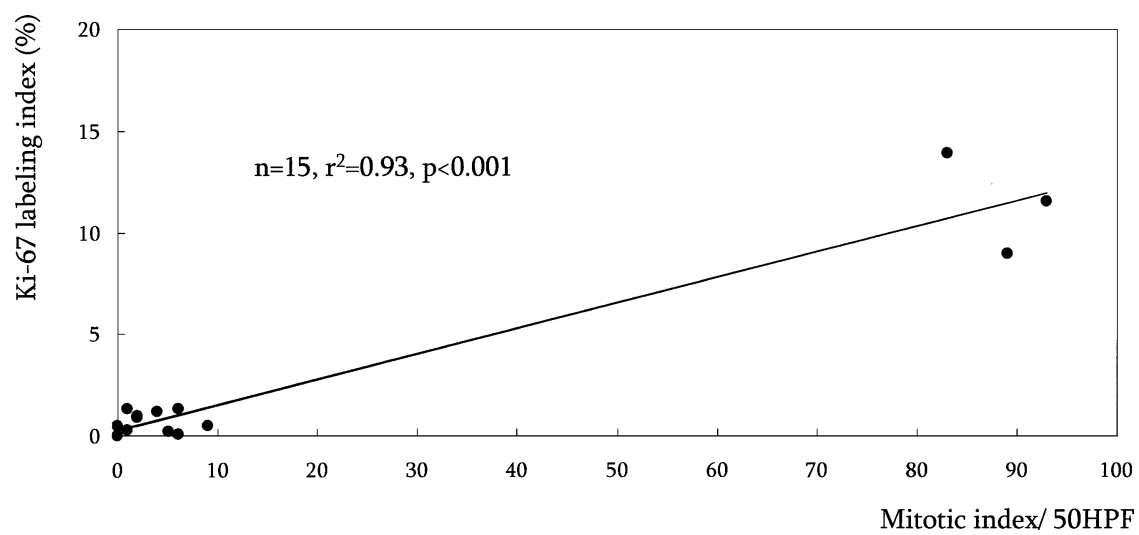

Fig. 1. Correlation of mitotic index and Ki-67 (MIB-1) labeling index. $H P F$, highpower fields

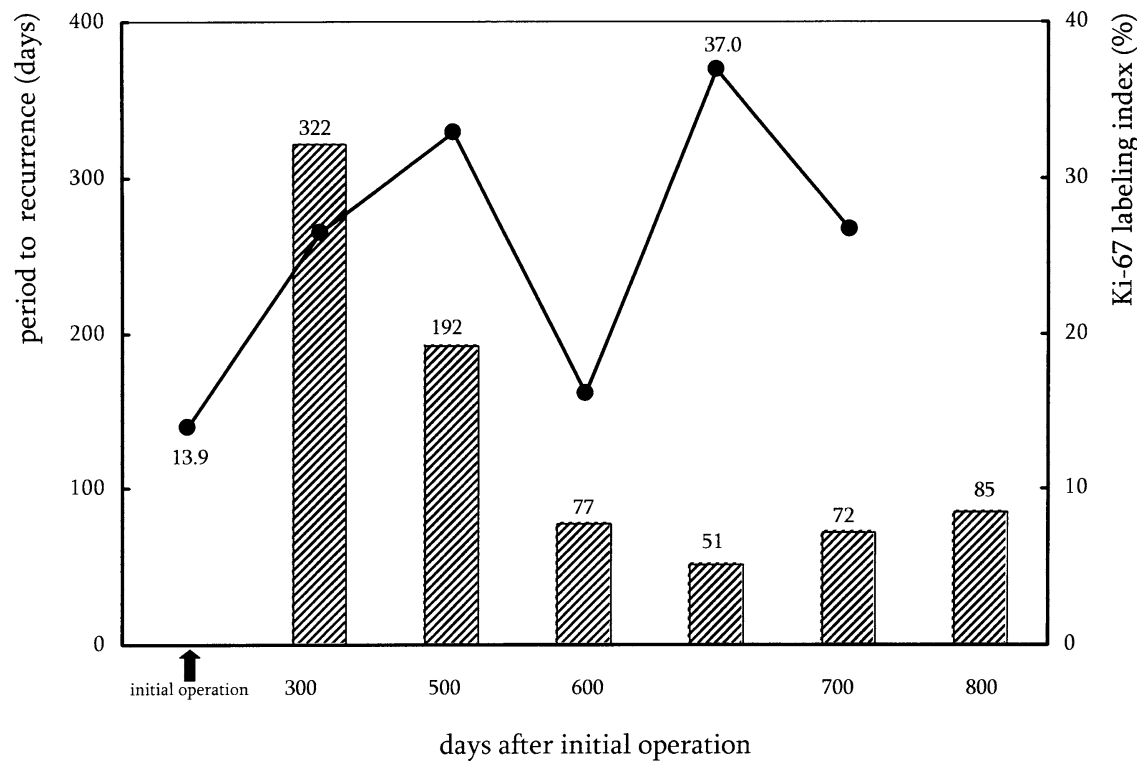

Fig. 2. Changes in malignancy in patient with peritoneal recurrence. Hatched bars show period to recurrence, and the solid line shows the Ki-67 labeling index nancy of GISTs. However, because patients with low values for tumor diameter or mitotic index are not necessarily disease-free after operation, longterm follow-up is essential. Rosai [2] classified tumors as "malignant", if the mitotic index value was 5/10 HPF or more and the tumor diameter was $5 \mathrm{~cm}$ or more. On the other hand, Amin et al. [3] classified tumors as "malignant" (mitotic index, $>5 / 50$ HPF) irrespective of the tumor diameter; "borderline" (mitotic index, $<5 / 50 \mathrm{HPF}$ and tumor diameter, $>5 \mathrm{~cm}$ ); and "benign" (mitotic index, $<5 / 50$ HPF and tumor diameter, $<5 \mathrm{~cm}$ ), preparing evaluation criteria based on the pattern of mitosis and the diameter of tumors. Shimoda et al. [4], who investigated 140 patients with gastric stromal tumors, reported that tumor diameter of more than $10 \mathrm{~cm}$, mitotic index of more than 10/200 HPF, and a Ki-67 labeling index of more than $10 \%$ were independent indicators of poor prognosis on multivariate analysis, and they found that the $\mathrm{Ki}-67$ labeling index was more than $10 \%$ in all of the patients with a mitotic index of more than 10/200 HPF. There is a problem of differences in the calculated frequencies of mitosis by various pathologists, and the interval until fixation is known to affect the frequency of mitosis. The Ki-67 labeling index, which is determined immunohistochemically by evaluating the cell growth-related antigen Ki-67, using the monoclonal antibody MIB-1, has recently been used as an index of cell growth, and the prognosis of GISTs was reported to be significantly poor when the Ki-67 labeling index was $10 \%$ or more [5]. The mitotic index reflects the $\mathrm{M}$ stage of mitosis only; but, because the Ki-67 labeling index can recognize most of the proliferating cells in stages G1, S, G2, it is considered to be more appropriate as an objective index of the malignancy of GISTs. In our investigation, the 3 patients in the metastasis/recurrence group all had a Ki-67 labeling index of $10 \%$ or more, significantly higher than the value in the other 12 patients, who were in the benign tumor group.

In general, the malignancy of GISTs in the stomach is known to be lower than that in the small intestine, if 


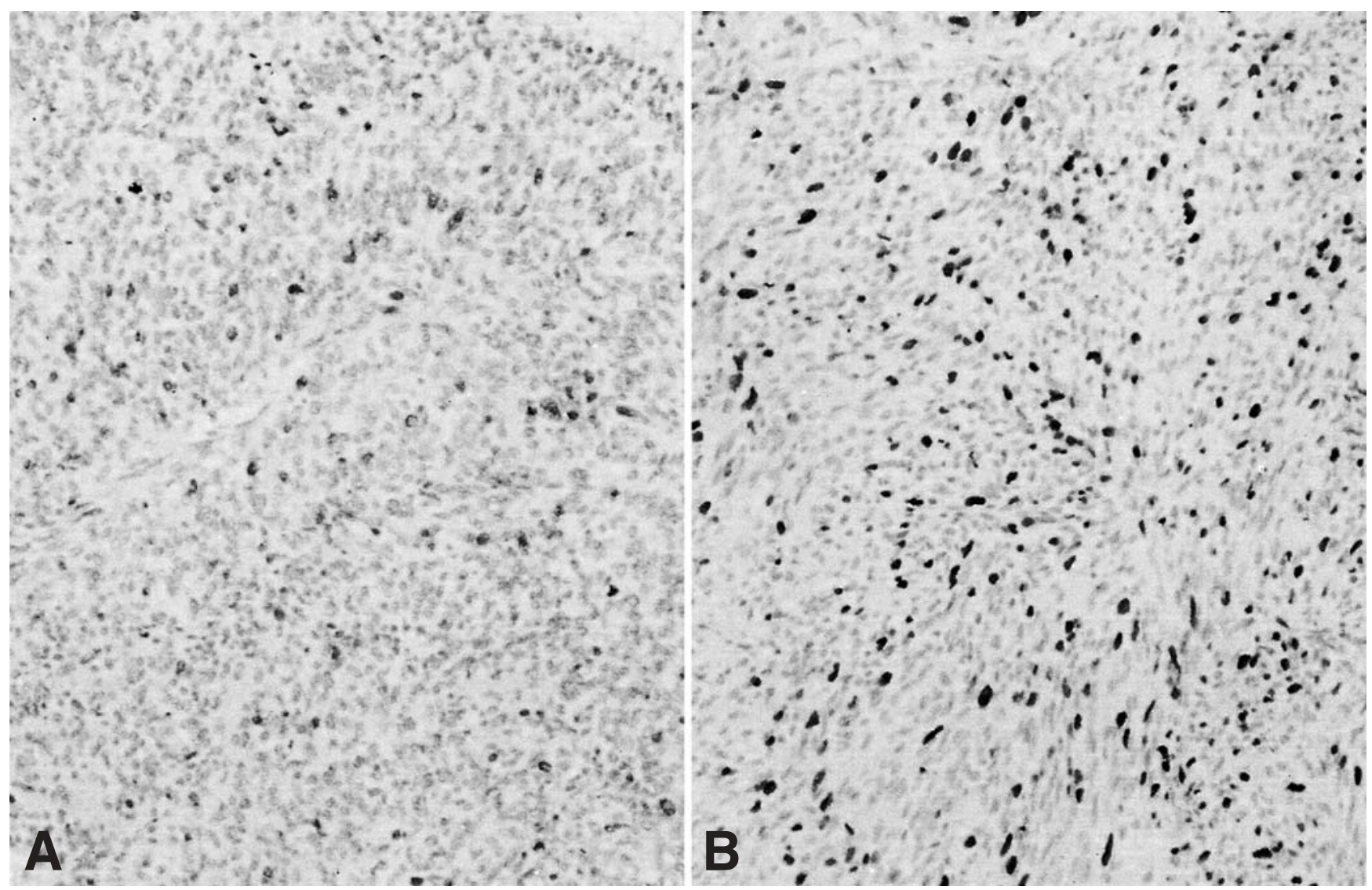

Fig. 3A,B. Changes in Ki-67 labeling index. A Value at the first operation, $13.9 \%$; $\mathbf{B}$ at the fifth operation, $37.0 \%$

there is no difference in the tumor diameter or the frequency of mitosis; the curative resection rate for gastric stromal tumor and the 5-year survival rate after curative resection have been reported to be $95 \%-99 \%$ and $75 \%-83 \%$, respectively [6-8]. Although most of the recurrences have been reported to occur in the liver $(40 \%-56 \%)$ or peritoneum $(11 \%-40 \%)$, no effective treatments have been established [9]. Unlike the prognosis of the primary GIST, that of peritoneal metastasis of gastric stromal tumor has been reported to be favorable, if lesions can be resected completely [10]. However, no conclusive evidence has been obtained for the efficacy of several operations because of recurrence to the peritoneum. Although some life-prolonging effect may be obtained, the efficacy of repeated surgical resection may be limited in terms of the burden on the patient and therapeutic efficacy. The patient we investigated had undergone five reoperations; finally she died of the primary disease, because of the metastasis to the liver ( 3.5 years after the initial operation) and because of the metastases to the peritoneum. In this patient, the interval to recurrence tended to decrease gradually, and the malignancy of the gastric stromal tumor, evaluated by the Ki-67 labeling index, was higher at the time of recurrences than at the time of the initial operation. Our impression was that the tumor became more brittle and was therefore more easily broken with the increasing number of reoperations, but the possibility cannot be ruled out that intraperitoneal scattering of tumor cells occurred due to surgical manipulation. Further, it also appears possible that surgical invasion has some influence on the DNA of tumor cells and that cytokines or the like, which are formed as an indirect effect, have a biochemical influence (e.g., by causing dedifferentiation), and this causes the gradual increase of the Ki-67 labeling index, as an index of malignancy.

It has recently been clarified that the autoactivation of tyrosine kinase due to the upregulation of the c-kit gene is related to the essential proliferation of GIST cells, and imatinib mesilate (STI-571), which inhibits the autoactivation of tyrosine kinase, has been reported to be effective as a molecule-targeting treatment [11]. Although the effects of this treatment still remain unclear with respect to the efficacy of prolonged use and adverse reactions, it is expected to be used as a first-line treatment in patients with unresectable gastric stromal tumors or patients with metastasis/recurrence. Because the efficacy of surgical resection alone is considered to 
be insufficient in patients with gastric stromal tumor with disseminated metastasis to the peritoneum, the outcome of operation is expected to be improved by the introduction of STI-571 in the future.

\section{References}

1. Hasegawa $T$, Yamamoto $S$, Nojima $T$, Hirose $T$, Nikaido $T$, Yamashiro $\mathrm{K}$, et al. Validity and reproducibility of histologic diagnosis and grading for adult soft-tissue sarcomas. Hum Pathol 2002;33:111-5.

2. Rosai J. Stromal tumors. In: Rosai J, editor(s) Ackerman's surgical pathology. 8th Ed. St. Louis: Mosby-Year Book; 1996. pp. 6457; pp. 691-3.

3. Amin MB, Ma CK, Linden MD, Kubus JJ, Zarbo RJ. Prognostic value of proliferating cell nuclear antigen index in gastric stromal tumors. Correlation with mitotic count and clinical outcome. Am J Clin Pathol 1993;100:428-32.

4. Shimoda T, Fujimoto Y, Hasegawa T, Nakanishi K. A concept and issue of GIST (gastrointestinal stromal tumor). Pathol Clin Med 2002;20:134-40.
5. Wang X, Mori I, Tang W, Utunomiya H, Nakamura M, Nakamura Y, et al. Helpful parameter for malignant potential of gastrointestinal stromal tumors (GIST). Jpn J Clin Oncol 2002;9:347-51.

6. Yuasa N, Takagi K, Oota H, Nishi M, Yanagisawa A. Clinicopathological examination of 76 cases of gastric leiomyoma. J Jpn Surg Soc 1992;93:248-56.

7. Sasako M, Katai H, Sano T, Noda N, Maruyama K, Ochiai A, et al. Selection of treatment for leiomyoma of the stomach. Stomach and Intestine 1995;30:1169-74.

8. Nashimoto A, Yabuzaki Y, Tanaka O, Oota T. Diagnosis and treatment for stromal tumor of the stomach - malignant GIST. Geka (Surgery) 2001;63:1051-7.

9. DeMatteo RP, Lewis JJ, Leung D, Muden SS, Woodruff JM, Brennan MF. Two hundred gastrointestinal stromal tumors. Recurrence patterns and prognostic factors for survival. Ann Surg 2000;231:51-8.

10. Emocy TS, Sobin LH, Lukes L, Lee DH, O'Leary TJ. Prognosis of gastrointestinal smooth-muscle (stromal) tumors. Am J Surg Pathol 1999;23:82-7.

11. Joensuu H, Roberts PJ, Sarlomo-Rikala M, Andersson LC, Tervahartiala P, Tuverson D, et al. Effect of the tyrosine kinase inhibitor STI571 in a patient with a metastatic gastric intestinal tumor. N Engl J Med 2001;344:1052-56. 